\title{
Public preferences regarding informed consent models for participation in population-based genomic research
}

\author{
Jodyn Platt, MPH ${ }^{1,2}$, Juli Bollinger, MS ${ }^{3}$, Rachel Dvoskin, $\mathrm{PhD}^{3}$, Sharon L.R. Kardia, $\mathrm{PhD}^{1,4}$ and \\ David Kaufman, $\mathrm{PhD}^{3}$
}

Purpose: Some large population biobanks that house biospecimens and health information for research seek broad consent from participants, whereas others reconsent for specific new studies. Understanding research participants' attitudes and preferences about broad and narrow consent may improve recruitment, retention, and public support.

Methods: An online survey was conducted among a representative sample of 4,659 US adults to examine relationships between consent preferences and demographic factors, beliefs about privacy and the value of research, and the perceived trustworthiness of researchers.

Results: Participants preferred broad consent (52\%) over studyby-study consent models (48\%). Higher preferences for study-bystudy consent observed among black non-Hispanic respondents and respondents with lower income and education were explained by differences in the prevalence of one or more beliefs about the study. Respondents with fears about research and those who would feel respected if asked for permission for each research use preferred study-by-study consent. Preference for broad consent was related to the desire not to be bothered with multiple requests and the belief that the study could lead to improved treatments, cures, and lives saved.

Conclusion: These data suggest that support for broad consent is contingent on sufficient information about data use. Work with research participants and community leaders to understand, respond to, and influence opinions about a given, ongoing study may improve uptake of broad consent.

Genet Med advance online publication 9 May 2013

Key Words: biobank; broad consent; informed consent; large population studies; public engagement

\section{INTRODUCTION}

The scientific and economic efficiencies offered by large data collections such as those proposed by the National Human Genome Research Institute (NHGRI); ${ }^{1}$ the VA Million Veterans Project; $^{2}$ the Kaiser Permanente Research Program on Genes, Environment and Health; ${ }^{3}$ and the UK Biobank ${ }^{4}$ promise to accelerate research from "bench to bedside". ${ }^{5}$ Biobanks catalog and store biological samples linked to individual-level health data for entire populations. ${ }^{6}$ Technological advances have created simpler, less-expensive ways to measure vast arrays of molecular data (e.g., genome-wide chips for DNA and RNA) and to catalog and store sensitive health information (e.g., barcoding, robotic retrieval, encryption, and firewalls). Historic longitudinal studies such as the Framingham Heart Study have added DNA sequencing components to their protocols. ${ }^{7}$ Researchers with large genotyped populations have routinely sought to link these genotype data to a wider range of phenotypes to address new research questions. Trends in scientific research toward the use of large-scale biobanks and long-term population-based longitudinal studies challenge the traditional role of informed consent as a social contract between a single research team and an individual participant.

Informed consent has generally operated under a model in which one subject agrees to participate in one study using a single consent form. However, when materials are collected and held indefinitely, with the intent of sharing data with outside researchers, the future uses of data and samples are not completely known, and, consequently, risks and benefits cannot be precisely defined at the point of consent. ${ }^{8}$ When participants are not informed about specifics of future studies using their samples, they are requested and expected to endorse a biobank's process of data management and to trust its ethical oversight and stewardship of information. ${ }^{9}$

From a biobanking policy perspective, three main options for opt-in consent seem plausible. First, broad or blanket consent would ask individuals to participate in unknown future research, with the recognition that future studies would be subject to ethical review. Second, study-by-study consent would entail researchers or biobanks approaching individuals on an ongoing basis to obtain consent for a specific research projects. Third, individuals might preselect the types of research they would like their information and samples to be used for. Broad and study-by-study consent models are among the most frequently used current policies and practices being used in longitudinal cohort studies and retrospective biobanks. ${ }^{10,11}$ If enacted, a proposed change to the Common Rule that would require consent for research using deidentified biospecimens could greatly increase the use of broad consent models. 
The choice of a consent model has important implications for biobanks and cohort studies. Although many researchers see the use of broad consent as a practical, efficient way of maximizing the research uses of collected data and samples, such models require forethought about what research activities will and will not be supported. In cases in which biobanks wish to share their data with the wider research community, policies on data sharing and privacy protection must be drafted that meet the requirements of institutional review boards and participants. As biobank managers and researchers consider which consent model to deploy, it may be useful to consider the perspectives of potential participants. Measuring the acceptability of broad consent as compared with a more traditional study-by-study method and examining the beliefs and opinions that underlie a reluctance to participate in studies using onetime consent for open-ended future uses may help researchers decide whether broad consent is indeed appropriate or how to address public concerns and improve recruiting of representative study samples. In light of litigation that has successfully challenged state public health departments' collection and use of newborn screening bloodspots and related health information, ${ }^{12}$ the question of what constitutes a clear, adequate, and acceptable consent process is relevant to all biobanks.

To inform the design of effective consent and concomitant participant engagement, it may be useful to measure potential research participants' preferences and explore the beliefs that lie behind them. For example, individuals with fears about the scope of the research process may be more inclined to participate in research if consent and ongoing engagement efforts provide detailed explanations of the types of work the biobank will and will not support. As part of a public consultation project to understand the conditions under which the general public might participate in a large population study proposed by NHGRI, a survey was administered to a representative sample of adults aged 18 years or older $(n=4,659) \cdot{ }^{13}$ Using the results of this survey, we examine the relationships between preferences for informed consent (broad versus study-by-study) and demographic characteristics including age, education, sex, race or ethnic group, income, and urban/ rural residency, as well as respondents' privacy concerns and their attitudes about research and researchers, including general trust of research, and general and specific concerns and values related to research participation. Because of the movement in the field toward broad consent models, the discussion addresses some of the issues and considerations our findings bring up for studies considering or using broad consent. However, this should not be construed as endorsement of broad consent. The study was not conducted to support the use of one consent model or another but to collect and interpret public opinions to inform study design and policy.

\section{MATERIALS AND METHODS}

The survey reported here was part of a larger study, conducted as a cooperative agreement with the NHGRI, to measure public attitudes about a hypothetical nationwide National Institutes of Health-sponsored cohort study of 500,000 participants to study gene-environment interactions contributing to common disease. To successfully launch and conduct a study recruiting from the diverse US population, public attitudes about such work were collected and reported. The survey followed a series of focus groups conducted across the United States. ${ }^{14}$

A 177-item online survey was developed to collect data on public opinions about a national cohort study proposed by the NHGRI and fielded between 14 December 2007 and 31 January 2008. A Web-based questionnaire, based on focus groups conducted nationwide in 2007, consisted of four main sections. ${ }^{13}$ Respondents answered questions about their health, values, and beliefs and then watched a 3-min video that described the goals and design of the proposed cohort study and that is available online. ${ }^{15}$ Following the video, participants answered questions about their interest in and concerns about the study. (For additional information about the survey content and design, see ref. 13 and ref. 15 provides a link to the video.)

The company Knowledge Networks (KN) managed sample selection and online administration of the survey. At the time, KN maintained a probability-based sample of $\sim 40,000$ US adults enrolled using random-digit dialing. For people who were interested in participating but lacked Internet access, $\mathrm{KN}$ provided a laptop/tablet and Internet access. KN sample members were routinely cycled out of the pool, and new members were brought in to avoid survey fatigue. Participants in KN's sampling pool were representative of the general population in terms of age, sex, race/ethnicity, and other core demographic and socioeconomic variables. ${ }^{16}$ During the field period, 8,735 potential respondents aged 18 years and older were randomly sampled from KN's Web-enabled master panel of US residents; the goal was 4,910 respondents, including a random sample of 3,700 and oversamples of 480 black non-Hispanics, 480 Hispanics, and 250 people living outside of metropolitan statistical areas. ${ }^{16,17}$ Poststratification weights corresponding to the US Census demographic benchmarks for age, sex, household income, education, and race and ethnic background were calculated by $\mathrm{KN}$ for this survey sample, to account for the oversamples and to reduce bias from sampling error. A separate set of poststratification weights was also created for each of the oversampled groups to enable analyses within each of these groups.

\section{Analysis methods}

The questionnaire included 22 Likert scales that measured attitudes about research and researchers both generally and in the context of the cohort study. Independent variables were grouped into one of four categories of factors that we expected would shape consent preferences: (i) trust, (ii) privacy, (iii) specific concerns about participating in the proposed NHGRI research project, and (iv) beliefs about the value of participating in research-i.e., whether it has social or personal benefit. The trust-related variables indicate a general trust (or mistrust) in researchers to be ethical. Privacy variables reflect an individual's general concerns about privacy infringements and the protection of medical data. We grouped specific concerns related to the NHGRI study in the third category. The fourth category 
captures the value that an individual places on research both generally and with respect to the proposed NHGRI study in a series of questions about potential personal or social benefits.

To evaluate collinearity within and across the four groups of variables, we examined correlations between variables before fitting the statistical models. Correlations were not as high as anticipated, but in instances for which the correlation was $>0.5$, we reviewed the questions and determined which to keep in the model based on which question seemed to most directly measure the attribute of interest. Three variables relating to feelings of respect and involvement in the study were ultimately dropped from the model. For the remaining 19 variables, correlations did not exceed 0.44 , and most were $<0.1$.

The outcome variable used to model consent preference was derived from 4,568 individuals' valid responses to the question, "In thinking about the consent you would give, would you prefer: to give your permission at the beginning of the study for all research projects that the oversight committee approves; to be asked for permission to use samples and information for each research project; [or] to preselect certain types of research from a menu of options." We call these options "broad consent," "studyby-study," and "menu," respectively. We included only those respondents who chose broad $(46 \%, n=1,704)$ or study-by-study $(44 \%, n=1,643)$ consent in the majority of our analyses because we were fundamentally interested in the differences between people who favored traditional study-by-study consent and broad consent. We came to this decision on the basis of three factors: first, a relatively small number of individuals $(10 \%, n=386)$ indicated preference for the "menu" option; second, as de facto policy options, broad and study-by-study are the most feasible, and differences between them are being widely discussed in the literature on biobank consent; and third, preliminary analyses suggested that the difference between characteristics of those who prefer menu consent and study-by-study consent is minimal.

Participants who preferred menu consent and those with missing information for any of the dependent or independent variables modeled $(n=835)$ were excluded from the analysis, leaving a sample size of 3,347. Distributions of age, income, education, race and ethnic background, and rural residency were nearly identical between the group included in the analysis and those excluded for missing data (all $P \geq 0.10$ ). Women were underrepresented in the group analyzed as compared with those excluded as missing ( 49 vs. $58 \% ; P<0.0001$ ). In the final sample, $52 \%$ preferred a broad consent model, as compared with $48 \%$ who preferred study-by-study consent.

A series of three weighted logistic regression models were fitted to predict the probability that an individual would opt for broad consent rather than study-by-study consent. In model 1 , we examined the effects of the demographic characteristics summarized in Table 1. In model 2, the demographics and 19 additional covariates were used (Table 2). Model 3 included only factors with $P$ values $\leq 0.10$ in model 2 . The Akaike information criterion was used to measure whether adding covariates to a model improved it or not. In this case, the Akaike

Table 1 Demographic variables considered in models 1 (demographic factors only), 2 (all covariates), and 3 (covariates with $P<0.10$ in model 2) $(n=3,347)$

\begin{tabular}{|c|c|c|c|c|c|c|}
\hline Demographic factor & \% (Unweighted) & $\begin{array}{c}\text { Favored a broad } \\
\text { consent } \\
\text { model }(\%)\end{array}$ & $\begin{array}{c}\text { Favored } \\
\text { study-by-study } \\
\text { consent }(\%)\end{array}$ & $\begin{array}{l}\text { Model } 1, \\
\text { odds ratio } \\
\text { ( } P \text { value) }\end{array}$ & $\begin{array}{l}\text { Model } 2, \\
\text { odds ratio } \\
\text { ( } P \text { value })\end{array}$ & $\begin{array}{c}\text { Model } 3, \\
\text { odds ratio } \\
\text { ( } P \text { value) }\end{array}$ \\
\hline Male & $50(51)$ & 57 & 43 & & & \\
\hline \multicolumn{7}{|l|}{ Race/ethnicity } \\
\hline White non-Hispanic ${ }^{\mathrm{a}}$ & $62(61)$ & 54 & 46 & & & \\
\hline Hispanic & $16(18)$ & 48 & 52 & $0.86(0.386)$ & $0.92(0.661)$ & - \\
\hline Other & $6(5)$ & 55 & 45 & $1.02(0.926)$ & $1.11(0.645)$ & - \\
\hline Did not complete BA $\mathrm{A}^{\mathrm{a}}$ & $73(70)$ & 51 & 49 & & & \\
\hline Completed BA degree & $27(30)$ & 57 & 43 & $1.14(0.176)$ & $0.95(0.683)$ & - \\
\hline Income $<\$ 75,000^{a}$ & $78(75)$ & 51 & 49 & & & \\
\hline Blood donation (no) ${ }^{\mathrm{a}}$ & $75(76)$ & 51 & 49 & & & \\
\hline Blood donation (yes) & $25(24)$ & 58 & 42 & $1.30(0.588)$ & $1.08(0.558)$ & - \\
\hline $10-$ Year increase in age & & & & $1.09(0.008)$ & $1.07(0.046)$ & $1.07(0.052)$ \\
\hline Overall & & 52 & 48 & & & \\
\hline
\end{tabular}

BA, bachelor of arts.

aReference category. 
Table 2 Relationships between consent preferences and various opinions and beliefs

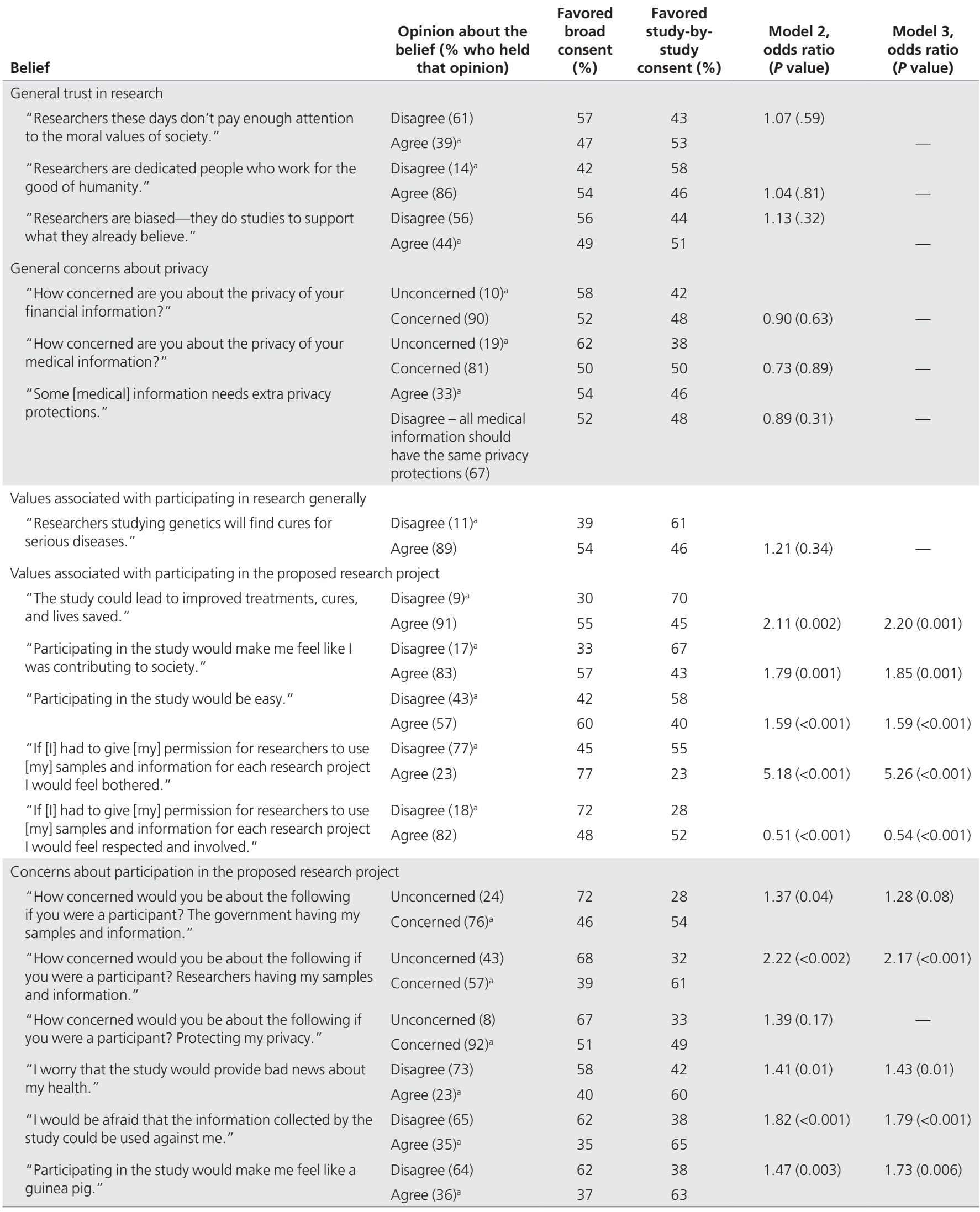

Odds ratios compare the odds of favoring broad consent between different belief groups.

aReference group or category. 
information criterion measures how well models discriminate between people who prefer broad and study-by-study consent, penalizing each model for the number of covariates it uses. The comparison between models 1 and 2 assesses whether adding all 19 opinion covariates improves on a model containing only demographic covariates. Comparing models 2 and 3 assesses whether including all possible covariates (model 2) improves on a model containing only covariates of borderline or better significance (model 3). The three models were run for the entire data set, as well as for men and women, because of the nonrandomness of missing data between the sexes; differences between sexes are noted below.

Marginal effects were calculated for the 12 variables in the final model. In this case, the marginal effect represents the percentage change in the support for broad consent that is associated specifically with a change in the value of one independent variable, adjusting for the other variables in the model. For example, looking in Table 3 at the belief that "the study could lead to improved treatments, cures, and lives saved," the marginal effect of +14.7 means that, on average, the probability that people who share this belief will choose broad consent is $14.7 \%$ points higher than it is for people who do not believe the study will lead to improved treatments, adjusted for other covariates in the model. This calculation simplifies the interpretation of the effect of the independent variables on the probability of choosing broad consent, making it comparable to interpretations commonly seen in linear models..$^{18,19}$

Regressions were performed using weights corresponding to US Census demographic benchmarks, to account for oversampling and to reduce bias from sampling error. A separate set of weights created for each of the oversampled groups enabled analyses within each of these groups.

\section{RESULTS}

In total, 7,978 people were contacted to take the survey, and 4,659 provided valid responses, for an overall response rate of $58.4 \%$. Weighted and unweighted demographic distributions of the complete survey sample were comparable to US Census figures for 2000. ${ }^{16}$ Table 1 lists weighted and unweighted distributions of demographic variables; the sample is split nearly evenly with respect to men and women; $61 \%$ are white, non-Hispanic; $16 \%$ are black, non-Hispanic; and 18\% are Hispanic; 70\% have less than a bachelor's degree education; 19\% live in a rural community; and three-quarters have annual household incomes $<\$ 75,000$. The group of 3,347 analyzed here was comparable to the entire surveyed population with respect to demographics except for sex; as described above, women were slightly but significantly underrepresented among those who qualified for this analysis. In this study sample, slightly more people preferred broad consent to study-by-study consent than in the overall sample.

\section{Predictors of a preference for broad consent over study-by- study consent}

The first model of demographic factors included age, sex, race/ ethnicity, education, urban or rural residency, and household income as predictor variables (Table 1). In model 1, older participants were significantly more likely to say they preferred broad consent, and women were less likely than men and black

Table 3 Marginal effects of independent variables in model 3

\begin{tabular}{|c|c|c|c|}
\hline Independent variables & $\begin{array}{l}\text { Marginal effect (percentage } \\
\text { points) (confidence interval) }\end{array}$ & $\begin{array}{l}\text { Sampling } \\
\text { error }\end{array}$ & $P$ value \\
\hline Sex (women, as compared with men)a & $-5.4(-9.4,-1.4)$ & 2.0 & 0.008 \\
\hline \multicolumn{4}{|l|}{ Values associated with participating in proposed research project } \\
\hline $\begin{array}{l}\text { If you had to give your permission for researchers to use your samples and } \\
\text { information for each research project: I would feel bothered }\end{array}$ & $30.5(25.8,35.2)$ & 2.4 & 0.000 \\
\hline Participating in the study would make me feel like I was contributing to society & $11.6(5.4,18.2)$ & 3.4 & 0.001 \\
\hline Participating in the study would be easy & $8.85(4.4,13.3)$ & & \\
\hline $\begin{array}{l}\text { If you had to give your permission for researchers to use your samples and } \\
\text { information for each research project: I would feel respected and involved }\end{array}$ & $-11.7(-17.4,-6.0)$ & 2.9 & 0.000 \\
\hline \multicolumn{4}{|l|}{ Concerns about participation in the proposed research project } \\
\hline Participating in the study would make me feel like a guinea pig & $-6.9(-11.9,-1.9)$ & 2.5 & 0.007 \\
\hline I worry that the study would provide bad news about my health & $-6.7(-11.8,-1.6)$ & 2.6 & 0.010 \\
\hline $\begin{array}{l}\text { Very or somewhat concerned about the government having my samples } \\
\text { and information }\end{array}$ & $-4.8(-10.3,0.7)$ & 2.8 & 0.086 \\
\hline
\end{tabular}

anterpretation of marginal effects for dummy variables (example): on average, the probability that a woman would choose broad over study-by-study consent is $5.4 \%$ points lower than it is for a man. 'Interpretation of marginal effects for continuous variables (example): on average, the probability of choosing broad over study-by-study consent increases $1.2 \%$ points for every 10 -year increase in age. 
Table 4 Specifications and fit of three tested models (lower AIC scores indicate preferable models)

\begin{tabular}{|c|c|c|c|}
\hline Model & $n$ & $\begin{array}{l}\text { Degrees } \\
\text { of } \\
\text { freedom }\end{array}$ & AIC \\
\hline Model 1: $\mathrm{P}(\mathrm{y}=$ broad $\mid \mathrm{X})$ & 3,347 & 9 & $4,591.97$ \\
\hline Model 2: P $(y=$ broad $\mid X T P R V)$ & 3,347 & 28 & $3,731.93$ \\
\hline $\begin{array}{l}\text { Model 3: } \mathrm{P}\left(\mathrm{y}=\text { broad } \mid \mathrm{X}_{1} T_{1} \mathrm{P}_{1} \mathrm{R}_{1} \mathrm{~V}_{1}\right) \\
(\alpha<0.10 \text { for inclusion in model 3) }\end{array}$ & 3,347 & 13 & $3,725.68$ \\
\hline
\end{tabular}

AIC, Akaike information criterion; $P$, general concerns about privacy; $R$, concerns about participation in proposed research project; $T$, general trust in research; $\checkmark$, values associated with participating in research; $X$, demographic factors; $1, \alpha=0.10$ in model 2 .

non-Hispanic participants were less likely than white nonHispanics to say they would prefer broad consent. The observed difference between Hispanics and white non-Hispanics was not significant after adjusting for other demographic factors.

In model 2, 19 variables related to general trust of research, general privacy beliefs, concerns related specifically to participating in the proposed NHGRI study, and beliefs about the value of research were added to the six demographic variables (Table 2); this resulted in a better specified model of participants' consent preferences than model 1 , based on the difference in Akaike information criterion values (Table 4). Model 3 , which included only factors with $P$ values $<0.10$ in model 2 , achieved a slightly better fit than model 2 using fewer explanatory variables (Table 4). The 12 variables included in model 3 and their marginal effects on preference for broad consent are listed in Table 3. All parameters in model 3 were highly significant.

\section{Demographic factors}

The only demographic characteristics that remained significant in models 2 and 3 were age and sex. Older individuals are more likely to prefer broad consent (odds ratio (OR) for 10-year age increase $=1.07 ; P=0.05)$ Overall, $54 \%$ of men and $48 \%$ of women chose broad consent over study-by-study consent. Sex remained a significant factor in the final model (model 3): adjusting for other variables in the model women were less likely than men to prefer broad consent $(\mathrm{OR}=0.75 ; P=0.008)$.

Women were significantly underrepresented in the sample of those with no missing data. To assess whether there were any major differences in the attitudes underlying men and women's preferences for broad consent, we stratified the sample by sex and examined the covariates in model 3 (data not shown). For both men and women, the two most important factors were the belief that study-by-study consent would make them feel bothered and concern about researchers having their samples and information. However, several differences were observed between men and women. The belief that "participating in the study would make me feel like a guinea pig" was significantly related to preference for study-by-study consent among women $(\mathrm{OR}=0.62$ for selection of broad consent; $P=0.01)$ but not among men $(\mathrm{OR}=0.77 ; P=0.15)$. The beliefs that study-by-study consent would make the participant feel more respected and involved and that participating in the study would be easy or would provide bad news about one's health were only significantly related to men's preferences.

\section{General trust of research, general privacy beliefs}

Beliefs about whether researchers uphold the moral values of society, whether they are dedicated people who work for the good of humanity, and whether they are biased were unrelated to consent preference. Likewise, none of the questions related to general beliefs about privacy were associated with a preference for consent, after adjusting for participants attitudes about the value of research and their specific concerns about the proposed study.

Beliefs about the value of research and concerns related specifically to participating in the proposed NHGRI study

With the exception of concern about protecting one's privacy in the study, all of the variables measuring values and concerns about participating in the NHGRI-proposed research study were significantly and independently associated with preference for the study-by-study consent model. The most important of these were concerns about researchers having information about them $(\mathrm{OR}=2.17 ; P=0.000)$ and fear that data collected by the study could be used against them $(\mathrm{OR}=1.79 ; P=0.000)$. Of note, fear of government having their data was not a significant predictor after adjusting for other factors $(P=0.083)$.

Several of the variables measuring values about research participation were associated with consent preference. Those who would feel respected and involved if they were asked permission for each research study strongly preferred study-by-study consent. Respondents who believed that participating would "make me feel like I was contributing to society," $(\mathrm{OR}=1.85 ; P=0.001)$ that the study would accelerate medical treatments and cures (OR $=2.20 ; P=0.001)$, or that participating in the cohort study would be easy $(\mathrm{OR}=1.59 ; P<0.001)$ were all significantly more likely to prefer broad consent. In addition, those who said they would feel bothered if they had to give permission for each study were also significantly more likely to support broad consent $(\mathrm{OR}=5.26$; $P<0.001)$. A general belief in the value or benefit of research to society, i.e., "researchers studying genetics will find cures for serious diseases" was not statistically significant $(P=0.346)$.

Table 3 shows the marginal effect of each belief on support for broad consent adjusting for the other factors in model 3. The greatest marginal effect is among those who indicated that they would feel bothered if they were asked permission for each research use. On average, the probability that someone would choose broad over study-by-study consent was $30 \%$ higher for those who said they would be bothered if asked each time as compared with those who said they would not. A preference for study-by-study consent was associated most strongly with the belief that study-by-study consent would make a person feel respected and involved, that researchers would have and could misuse samples and information, and that the "information collected by the study could be used against me." 


\section{DISCUSSION}

Overall, given a choice, more survey respondents would opt for broad consent over a study-by-study consent model, particularly if they are confident in or supportive of the details of the study. We hypothesized that general trust in the research enterprise, general concerns about privacy, and concerns and perceived values specific to participation in the study research would be associated with preferences for broad or study-bystudy consent models. Of note is that after people's feelings about the value of research and their concerns about participation in the proposed NHGRI research project were accounted for, more general concerns about research and privacy beliefs did not significantly influence consent preferences. This suggests that researchers may have the opportunity to influence acceptance of broad consent if they understand and can address potential participants' study-specific concerns. This finding is consistent with Hoeyer's literature review ${ }^{20}$ that found that the type and context of a study has implications for informed consent policy choices.

As broad consent continues to be considered by researchers and recommended by research policymakers, it will be important to understand how to implement it in ways acceptable to the public. We are not trying to promote the broad consent approach but rather to discuss how our findings might be useful to those who are applying or considering broad consent methods. One example is the relevance of issues on privacy and trust. Participants' general concerns about privacy and trust in research were not related to consent preferences when controlling for other variables. By contrast, the factors associated with a preference for study-by-study consent basis reflect specific concerns about the proposed NHGRI study, such as who would have access to the data and how that data could be misused to harm or disrespect a participant. This finding suggests that for studies contemplating the use of broad consent, actions that build trust such as communicating details about research policies and procedures could provide transparency and examples of trustworthiness.

Preference for broad consent is also associated with valuing the conduct of streamlined research that would be easy for participants and that might increase the potential for findings to treat or prevent disease and perhaps contribute to participants' feelings of accomplishment. On the other hand, the positive feelings of involvement and respect that being asked for consent would confer were strongly related to preference for study-bystudy consent.

Although several demographic covariates, including race and ethnic group, income, and education, initially appeared to be related to consent preference, these associations were no longer significant when beliefs were added to the model, indicating confounding with the demographic factors. If beliefs that dissuade people from broad consent cannot be addressed in these groups, studies using broad consent may have trouble recruiting in these segments of the population. Moreover, even after accounting for their beliefs, women and younger respondents were significantly less likely to choose broad consent. Studies using broad consent need to pay particular attention to these groups.

However, to the extent that researchers can work with potential participants and community leaders to pursue "active trust" ${ }^{\prime 21,22}$ by informing and responding to specific concerns and opinions about a given study, our data suggest that acceptance of broad consent may improve. As details of data use and the types of studies conducted using biobank or longitudinal cohort study samples evolve over time, studies using broad consent may also need to consider ongoing communication efforts to ensure trust and public support, given participants' interests in specific data uses and governance. As the Department of Health and Human Services considers rule changes that would encourage or require the use of brief, broad consent forms for research on broader categories of biospecimens, researchers may need to consider the development of supplementary materials (such as a Web page or Frequently Asked Questions document) that would promote transparency and address the questions for those who have them.

Given the broad digital access in the United States and the cost effectiveness of electronic outreach, ${ }^{23}$ Internet-based solutions including the use of social media could improve researchers' communication and increase transparency, as well as research participants' autonomy and control of their preferences. ${ }^{24}$ Investment in electronic infrastructure and online recruitment could make various consent models easier to implement and maintain over time. Online access would allow research participants to monitor use of their stored data and samples, potentially allaying fears of misuse while demonstrating responsible stewardship to research participants. ${ }^{25}$

\section{Limitations}

The goal of our analysis was to identify correlates to preferences for broad and study-by-study consent, assuming the respondent would participate in the cohort study. The order of questions and information in the survey could have influenced choices about how a respondent answered questions. For example, questions about specific study concerns (concern about protecting privacy, about government and researchers having samples and information) followed after participants were asked to choose their consent preference. With a preference already chosen, participants might have responded to these attitudinal questions differently than if they had not been afforded this choice.

We did not examine preferences among actual biobank participants; survey participants were members of the general public. We did not examine in detail the differences between those who preferred broad or narrow consent and those who would prefer menu-based consent. However, preliminary analyses indicated that people supporting menu consent shared beliefs with those who preferred study-by-study consent. As menu consent becomes a more feasible and practicable way of managing consent, preferences for menu consent should be explored further. The survey also focused specifically on a proposed, federally-funded, genetic study; the relevance of these findings to 
other longitudinal cohort studies and biobanks in general warrants additional investigation. Further systematic study of the attitudes of participants in these types of studies would address the generalizability and limitations of these findings. Perhaps more important, studies of such research participants will help to measure whether particular practices used in consent, community engagement, or study communication processes can successfully address some of the worries that appear to detract from the acceptability of broad consent models.

\section{Conclusion}

This analysis suggests that the current trend toward broad consent in genetic biomedical research may meet with acceptance similar to study-by-study consent so long as processes are evaluated and continually improved. Of those who chose either broad or study-by-study consent, a narrow majority preferred broad consent. Those with greater fears about research and those who would feel respected if they were asked for permission for each study prefer a model that allows participants to exercise a greater degree of autonomy by responding to specific requests for data usage. Factors associated with a preference for broad consent included the desire not to be bothered with multiple requests and the belief that the study could lead to improved treatments, cures, and lives saved. We found education, income, urban/rural residency, race/ethnicity, and the measures of privacy were not significant predictors of consent preferences after adjusting for beliefs and attitudes. Women and younger participants were more likely to prefer study-bystudy consent. General concerns about privacy and the research endeavor were unrelated to consent preference.

The ability to communicate, operate transparently, and demonstrate active respect for research participants appear to be fundamental to robust consent and research enterprises. In all large genomic studies, and particularly those using broad consent, participant and community engagement that can be done before and across the life of biobank-based resources should be considered. Engagement work that measures, acknowledges, and incorporates participant perspectives, or that communicates changes in the intent of the ongoing study and findings of the research could be a valuable tool in the recruitment and retention of participants. The practicality and acceptability of electronic and Web infrastructures to support the contact of participants in biobanks and long-term cohort studies according to their preferences could be explored as an avenue for making both models accessible.

\section{ACKNOWLEDGMENTS}

This work was funded by the National Human Genome Research Institute under grant number 1 U01 HG004206-0, Public Consultation to Inform the Design of Possible Large-Scale Studies of Genes and Environment in Common Disease. The authors thank Joan Scott of National Coalition for Health Professional Education in Genetics (NCHPEG) for her helpful input. Funding for the contributions of J.P. and S.L.R.K. came from the National Institute of Child Health and Human Development, grant number
5-R01-HD-067264-02, Linking Community Engagement Research to Public Health Biobank Practice.

\section{DISCLOSURE}

The authors declare no conflict of interest.

\section{REFERENCES}

1. Collins FS, Manolio TA. Merging and emerging cohorts: necessary but not sufficient. Nature 2007; 445(7125):259.

2. Veterans Health Administration. Million Veteran Program (MVP). Million Veteran Program (MVP). http://www. research.va.gov/mvp/. Accessed 16 August 2012.

3. Kaiser Permanente. The Research Program on Genes, Environment, and Health. http://www.dor.kaiser.org/external/DORExternal/rpgeh/index.aspx. Accessed 16 August 2012.

4. UK Biobank. Web Page. http://www.ukbiobank.ac.uk/. Accessed 16 August 2012.

5. Altshuler D, Daly MJ, Lander ES. Genetic mapping in human disease. Science 2008;322:881-888.

6. Yuille $M$, van Ommen GJ, Bréchot $C$, et al. Biobanking for Europe. Brief Bioinformatics 2008;9:14-24.

7. Splansky GL, Corey D, Yang Q, et al. The Third Generation Cohort of the National Heart, Lung, and Blood Institute's Framingham Heart Study: design, recruitment, and initial examination. Am J Epidemiol 2007;165:1328-1335.

8. Knoppers BM, Avard D, Cardinal G, Glass KC. Children and incompetent adults in genetic research: consent and safeguards. Nat Rev Genet 2002;3(3):221-225.

9. Caulfield T, McGuire AL, Cho M, et al. Research ethics recommendations for whole-genome research: consensus statement. PLoS biology 2008;6(3):e73.

10. Fullerton SM, Anderson NR, Guzauskas G, Freeman D, Fryer-Edwards K. Meeting the governance challenges of next-generation biorepository research. Sci Trans/ Med 2010;2:15cm3.

11. Solbakk JH, Holm S, Hofmann B. The Ethics of Research Biobanking. Springer: Dordrecht; New York, 2009:358. http://public.eblib.com/EBLPublic/PublicView. do?ptil $D=478061$

12. Rothwell $E$, Anderson R, Goldenberg A, et al. Assessing public attitudes on the retention and use of residual newborn screening blood samples: a focus group study. Soc Sci Med 2012;74(8):1305-1309. http://www.sciencedirect.com/ science/article/pii/S0277953612001050.

13. Kaufman D, Murphy J, Scott J, Hudson K. Subjects matter: a survey of public opinions about a large genetic cohort study. Genet Med 2008;10:831-839.

14. Murphy J, Scott J, Kaufman D, Geller G, LeRoy L, Hudson K. Public perspectives on informed consent for biobanking. Am J Public Health 2009;99:2128-2134.

15. Genetics and Public Policy Center. GPPC Proposed study video. http://www. youtube.com/watch?v=m-x81nkCP8A. Accessed 13 March 2013.

16. Knowledge Networks. Validity of the survey of health and internet and Knowledge Network's panel and sampling, 2003. http://www.cdc.gov/PCD/ issues/2004/oct/pdf/04_0004_01.pdf.

17. Kaufman DJ, Murphy-Bollinger J, Scott J, Hudson KL. Public opinion about the importance of privacy in biobank research. Am J Hum Genet 2009;85:643-654.

18. Anderson $S$, Newell RG. Simplified marginal effects in discrete choice models. Economics Letters 2003;81(3):321-326.

19. Cameron AC, Trivedi PK. Microeconometrics Using Stata. 2, Revised, Illustrated. Stata Press: College Station, TX, 2010:706.

20. Hoeyer K. Donors perceptions of consent to and feedback from biobank research: time to acknowledge diversity? Public Health Genomics 2010;13:345352.

21. Giddens A. The Consequences of Modernity. Stanford University Press: Stanford, CA, 1991:188.

22. Giddens A. Beyond Left and Right: The Future of Radical Politics. Stanford University Press: Stanford, CA, 1994.

23. Platt J, Platt T, Thiel D, Kardia S. 'Born in Michigan? You're in the biobank:' engaging population biobank participants through Facebook advertisements. Public Health Genomics, in press.

24. Zickuhr K, Smith A. Digital Differences, 2012. http://pewinternet.org/ Reports/2012/Digital-differences/Overview.aspx.

25. Thiel D, Platt J, Liebert J, King S, Kardia SLR. Town Hall and Online Responses to Informed Consent Models for Public Health Biobanks. Exploring the ELSI Universe: 2011 Congress. National Human Genome Research Institute, Ethical Legal and Social Implications Program. Chapel Hill, NC. 12-14 April 2011. 